\title{
Reconstructing Small Perturbations of an Obstacle for Acoustic Waves from Boundary Measurements on the Perturbed Shape Itself
}

\author{
Habib Zribi ${ }^{1}$ \\ ${ }^{1}$ University of Hafr Al Batin,
}

July 26, 2020

\begin{abstract}
We derive relationships between the shape deformation of an impenetrable obstacle and boundary measurements of scattering fields on the perturbed shape itself. Our derivation is rigourous by using systematic way, based on layer potential techniques and the field expansion (FE) method (formal derivation). We extend these techniques to derive asymptotic expansions of the Dirichlet-to-Neumann (DNO) and Neumann-to-Dirichlet (NDO) operators in terms of the small perturbations of the obstacle as well as relationships between the shape deformation of an obstacle and boundary measurements of DNO or NDO on the perturbed shape itself. All relationships lead us to very effective algorithms for determining lower-order Fourier coefficients of the shape perturbation of the obstacle.
\end{abstract}

\section{Hosted file}

scattering[21-06-2020].pdf available at https://authorea.com/users/346206/articles/472321reconstructing-small-perturbations-of-an-obstacle-for-acoustic-waves-from-boundarymeasurements-on-the-perturbed-shape-itself 